\title{
Preface to the Special Issue: Computational approaches for the modeling of complex crack path and patterns
}

\author{
Michael Kaliske • Anna Pandolfi
}

Published online: 16 December 2019

(C) Springer Nature B.V. 2019

This Special Issue (SI) of International Journal of Fracture has been inspired by a recursive co-presence in several Computational Mechanics meetings of the two Guest editors, Michael Kaliske and Anna Pandolfi, at the time serving as coordinators of the Computational Mechanics groups in Germany and in Italy, respectively. For a long time, Computational Mechanics has been representing a formidable tool to study fracture, especially in the conditions of complexity, where theoretical approaches fail to provide a sound support to the analysis of experimental results.

In particular, in brittle or quasi-brittle materials under complex loading condition, the nucleation and propagation of cracks are still not completely understood and are under extensive investigation. The key issue is the difficulty to predict the crack pattern, when multiple loads are acting or the material is heterogeneous, anisotropic or damaged, and fracture is expected to manifest unstable behaviors. In principle, the propagation of cracks is driven by physical criteria expressing the local attainment of the ultimate strength of the material, which can be difficult to assess in real materials.

Recent formulations based on energetic reasoning opened an interesting avenue for the definition of new algorithms. Popular numerical techniques are based

M. Kaliske ( $\square)$

Technische Universität Dresden, Dresden, Germany

e-mail: michael.kaliske@tu-dresden.de

A. Pandolfi

Politecnico di Milano, Milan, Italy

e-mail: anna.pandolfi@polimi.it on finite element discretizations, where fracture is described explicitly by reproducing newly created surfaces. Surfaces are inserted between solid elements, or cross solid elements. Phase field approaches are attracting attention for the ease of the implementation. Boundary element techniques have been also used with satisfactory results. Recent approaches disregard the presence of a classical mesh, avoiding the complication of disconnecting volume elements. Advanced numerical models of fracture are based on multi-scale descriptions and in some cases integrate different numerical techniques.

In this special issue, we collect the contributions of researchers and scientists that are working on advanced numerical algorithms to explore possible future developments of numerical methods for crack nucleation and crack tracking. All the papers are characterized by innovative computational aspects that can be of benefit to the computational mechanics community.

Qinami et al. investigate approaches to improve the reliability of crack pattern predictions by a variational eigenfracture formulation. An r-adaptive method based on configurational forces and an h-adaptive quad-tree algorithm are compared and evaluated.

The contribution of Fischer and Marigo studies the use of gradient damage models in dynamic fragmentation. To understand the structural response, a stability analysis is carried out by examining the growth of perturbations. The number of fragments obtained depends 
strongly on the characteristic length of the nonlocal damage approach.

The work by Allix et al. focuses on the numerical difficulties observed when explicit time integration approaches are combined with damage models that suffer of mesh-dependency, and proposes to use bounded rate approaches that are able to overcome the issue at the constitutive level, and allow to conduct quasi-static damage simulations up to failure.

The failure of long fiber-reinforced composites is strongly determined by crack propagation in the matrix and the fiber matrix-interface on the microscale. These phenomena are numerically studied by a combination of the phase field approach and the cohesive zone model in the article by Guillen-Hernandez et al.

The contribution by Shabir et al. studies crack propagation in polycrystalline specimens by means of a generalized finite element method, where the grains are linear elastic isotropic and the boundaries between grains are cohesive. The adopted brittleness criterion depends on the cohesive law parameters and on the average grain boundary length, and leads to the definition of master load-displacement curve that generalizes the behavior of all the specimens with the same microstructure. This properties can be exploited to save computational time.

Sridhar and Keip propose a phase field approach to model crack propagation in a coupled electromechanical setting, accounting for anisotropy by means of structural tensors. They use a non-associative dissipative framework, where the fracturing processes are driven by the mechanical part of the coupled electromechanical driving force. Appropriate choices of degradation functions allow for the accommodation of various electrical boundary conditions along cracks.

We thank all the contributors for their work, that is at the forefront of the modern computational fracture mechanics, and hope that the reader will enjoy learning new algorithms and new approaches and may get new ideas for further developments.

Publisher's Note Springer Nature remains neutral with regard to jurisdictional claims in published maps and institutional affiliations. 